\title{
US veterans and their unique issues: enhancing health care professional awareness
}

\author{
This article was published in the following Dove Press journal: \\ Advances in Medical Education and Practice \\ I December 2015 \\ Number of times this article has been viewed
}

\section{Maria Olenick' \\ Monica Flowers' \\ Valerie J Diaz ${ }^{1,2}$}

'Nicole Wertheim College of Nursing and Health Science, Florida International University, Miami, FL, USA; ${ }^{2}$ Operational Health Support Unit Jacksonville, United States Navy Nurse Corps, Jacksonville, FL, USA

Correspondence: Maria Olenick Nicole Wertheim College of Nursing and Health Sciences, Florida international University, I 1200 8th Street, AHC3 - 329, Miami, FL 33199, USA

Tel + I 3053487757

Fax +I 3053487765

Emailmolenick@fiu.edu
Abstract: United States veterans are a multifaceted population with a distinct culture that includes, but is not limited to, values, customs, ethos, selfless duty, codes of conduct, implicit patterns of communication, and obedience to command. Veterans experience mental health disorders, substance use disorders, post-traumatic stress, and traumatic brain injury at disproportionate rates compared to their civilian counterparts. Eighteen to 22 American veterans commit suicide daily and young veterans aged 18-44 are most at risk. Health care professionals must be aware of patients' military history and be able to recognize suicide-risk factors, regardless of age. Advancement in medical technology has allowed servicemen to survive their injuries but, for many, at the cost of a traumatic limb amputation and associated mental scarring. Health care professionals must be able to address physical safety concerns, as well as, emotional health of veterans. Approximately 49,933 American veterans are homeless and face the same difficulties as non-veterans in addition to service-related matters. Separation from military service and issues related to complex multiple deployments are among specifically identified veteran issues. Successful veteran reintegration into civilian life rests upon providing veterans with training that builds on their military knowledge and skill, employment post-separation from service, homelessness prevention, and mental health programs that promote civilian transition. Preparing health care providers to meet the complex needs of a vast veteran population can be facilitated by implementing veteran content into curricula that includes veteran patient simulations and case studies, and utilizes veteran clinical faculty.

Keywords: veterans, veteran health care, veteran health issues, veteran content

\section{Introduction}

United States veterans are multifaceted and may be considered a population, a culture, and a subculture. Military culture includes, but is not limited to, the values, customs, traditions, philosophical principles, ethos, standards of behavior, standards of discipline, teamwork, loyalty, selfless duty, rank, identity, hierarchy, ceremony and etiquette, cohesion, order and procedure, codes of conduct, implicit patterns of communication, and obedience to command (LD Purnell, University of Delaware and Florida International University, personal communication, January, 2015). ${ }^{1}$

The American veteran population is a unique population. Varying military service branches and varying military experiences among the veteran population is unique. Varying wartime eras and health-specific issues associated with those eras are unique among the veteran population. From a comparison of veterans from the Vietnam, Persian Gulf, and Iraq/Afghanistan (Operation Iraqi Freedom [OIF]/Operation Enduring Freedom $[\mathrm{OEF}]$ ) war eras, Fontana and Rosenheck ${ }^{2}$ noted distinct differences. OIF/OEF 
veterans include fewer African-Americans, more Latinos, and more females than other eras. In addition, this group is younger, less likely to be married, less likely to have been incarcerated, and more likely to be gainfully employed. ${ }^{2}$ It was also observed that OIF/OEF veterans appear to be more socially integrated, less often diagnosed with substance abuse disorders, and required less Veterans Affairs (VA) disability compensation for post-traumatic stress disorders (PTSDs) when compared to their Persian Gulf and Vietnam veteran counterparts. $^{2}$

\section{US veteran-specific health issues Mental health or behavioral adjustment disorders}

Medical records of veterans reveal "that one in three patients was diagnosed with at least one mental health disorder $-41 \%$ were diagnosed with either a mental health or a behavioral adjustment disorder". ${ }^{3}$ In compensation or in combination with military-related diseases, many veterans develop substance use disorders (SUDs) and a large number ultimately commit suicide. LeardMann et $\mathrm{al}^{4}$ found that male veterans diagnosed with "depression, manic-depressive disorder, heavy or binge drinking, and alcohol-related problems" were significantly associated with an increased risk of suicide. Thus, identifying and treating mental health illness has the greatest potential to mitigate suicide risk. Unfortunately, reluctance to seek help or treatment makes diagnosing and treating mental illness difficult in this population.

\section{SUDs}

The stressors of military service increase the risk of veterans having problems with alcohol, tobacco, or drugs (or a combination). Johnson et $\mathrm{al}^{5}$ found that cigarette smoking and alcohol consumption is higher among veterans than non-military personnel. For some veterans, treatment of a comorbid condition (eg, PTSD, depression, pain, insomnia) may resolve the problem. For others, long-term care is required. Thus, multiple clinical practice guidelines have been developed "and evidence-based screening tools to help clinicians identify veterans with SUDs and improve outcomes". 5

\section{PTSD}

Also known as "shell shock" or "combat fatigue", PTSD results from witnessing or experiencing (directly or indirectly) a traumatic event. ${ }^{6}$ The disease is not limited to veterans, however, military personnel experience PTSD almost four fold ( $8 \%$ of non-military men versus $36 \%$ of male veterans)..$^{5}$ PTSD is an amalgam of symptoms, severity, and duration. According to the American Psychiatric Association, ${ }^{6}$ diagnosis is based upon four symptom categories: intrusive symptoms (flashbacks), avoidance of reminders (isolation), negative thoughts and feelings ("no one can be trusted"), and arousal and reactivity symptoms (exaggerated startle response). PTSD is often associated with "traumatic brain injury (TBI), military sexual trauma (MST), sleep problems, substance use, pain, and other psychiatric disorders, and requires comprehensive assessment" ${ }^{5}$ Treatment is aimed at therapy (psychotherapy, prolonged exposure therapy, family/ group therapy, and others), social support, and/or medication such as antidepressants. Screening tools and evidence-based guidelines have been developed to accurately and expeditiously assess and treat veterans.

\section{TBI}

TBI is "a traumatically induced structural injury and/or physiological disruption of brain function as a result of an external force". 5 TBI can be classified as mild, moderate, or severe depending on the length of unconsciousness, memory loss/ disorientation, and responsiveness of the individual following the event (ie, are they able to follow commands). While mild TBI (or concussion) is the most common, diagnosis is difficult since symptoms include "headaches, dizziness/ problems walking, fatigue, irritability, memory problems and problems paying attention". 5,7

\section{Depression}

Among the available data from the National Alliance on Mental Illness (NAMI), ${ }^{2}$ depression ranks among the most common mental health disorders. The diagnosis rate for veteran depression is 14\% (although NAMI believes depression is under diagnosed). Notably, $\mathrm{NAMI}^{2}$ found that individuals with PTSD were less likely to commit suicide versus those with depression probably due to the increased awareness and acceptance of PTSD. Despite its devastating effects, major depression is a treatable illness with $80 \%-90 \%$ success rate using medication, psychotherapy, and/or electroconvulsive therapy. ${ }^{2}$ Models of care, such as Translating Initiatives for Depression into Effective Solutions, show eight out of ten veterans are effectively treated. ${ }^{8}$

\section{Suicide}

With 18 to 22 veterans committing suicide on a daily basis, risk assessment and intervention are paramount. ${ }^{9}$ Private and public health care professionals must be aware of patients' military history (since not all veterans seek care in VA clinics $)^{5}$ and be able to recognize suicide-risk factors, 
regardless of age. Young veterans aged 18-44 years are most at risk of suicide; yet, Kemp and Bossarte ${ }^{9}$ found that even older veterans, aged 50 years and older, were still almost twice as likely to commit suicide versus non-veterans $(69 \%$ and $37 \%$, respectively). Additionally, " $11 \%$ of veterans who survive a first suicide attempt will reattempt within 9 months, and $6 \%$ of those will die". ${ }^{5}$ Kemp and Bossarte ${ }^{9}$ found evidence supporting the efficacy of VA health care systems in lowering veterans' non-fatal suicide attempt rate, thus referral to a VA facility is recommended for appropriate counseling and health services.

\section{Chronic pain}

With $82 \%$ of OEF and OIF veterans reporting chronic pain, diagnosis and treatment are essential. ${ }^{5}$ A comprehensive assessment of pain is crucial, but also identifying associated physiological/biological and psychological factors since "chronic physical pain is often associated with co-morbid conditions, including TBI and PTSD, that may complicate treatment". 5,7 Treatment should focus on concurrently addressing all conditions, with extreme cautionary use of opioids due to the heightened risk of veterans developing SUDs.

\section{Amputations}

Advancement in medical technology and bodily protection allow soldiers to survive injuries at a higher rate than in previous wars. Yet, the scars from a traumatic amputation are deep and many soldiers develop mental health injuries related to the event and "in cases involving multiple limb amputations or disfigurement, body image issues may create multiple social and employment barriers". 5 According to military casualty statistics, 1,573 veterans have suffered major loss of limb amputations from battle injuries since $2010 .^{10}$

Health care professionals must be able to address the physical safety concerns, as well as, the emotional health of the veteran. Sensory aids, prosthesis, and medical rehabilitation require an interdisciplinary-team approach in healing wounded soldiers.

\section{Rehabilitation care}

Many veterans have a hard time reacclimating into society after deployment due to military skills that are not transferrable to civilian life, bodily trauma that rendered that individual handicapped, and/or war-related mental disease. Rehabilitation care is aimed at a balance of vocational, physical, social, and mental therapies to prepare veterans for re-entry into civilian life. Vocational programs help job-seeking veterans develop skills and knowledge required for a particular job. Physical rehabilitation focuses on improving veterans' quality of life and independence. Social rehabilitation assists veterans to assimilate to non-military life and establish new ways of life post-deployment. Mental rehabilitation teaches veterans with mental health illness the living skills of community functioning and ability to deal with their new environment.

\section{Hazardous exposures}

Veterans' past exposure to chemicals (Agent Orange, contaminated water), radiation (nuclear weapons, X-rays), air pollutants (burn pit smoke, dust), occupational hazards (asbestos, lead), warfare agents (chemical and biological weapons), noise, and vibration increase their risk of health problems even years after the initial assault. ${ }^{11}$ For example, long-term health problems have been implicated in association with Agent Orange exposure in Vietnam veterans. ${ }^{12}$ For those who served in Iraq and Afghanistan, there is insufficient data to identify long-term health effects of hazardous exposure to pollutants, such as "burn pits" and infectious agents such as rabies, despite the immediate side-effects experienced by most veterans. ${ }^{5}$ Obtaining an accurate medical and deployment history is essential in providing accurate diagnosis and appropriate treatment.

\section{Homelessness}

It is estimated that approximately 49,933 veterans are homeless ( $12 \%$ of homeless adult population). ${ }^{13}$ Homeless veterans face the same difficulties as non-veterans such as substance use, unemployment, and mental illness; yet plagued with the additional burdens of military-related factors, "such as PTSD, TBI, a history of multiple deployments, and military skills that might not be transferable to the civilian work environment". National Coalition for Homeless Veterans ${ }^{13}$ found that $51 \%$ of homeless veterans have disabilities, 50\% suffer from a serious mental illness, and 70\% have SUDs. National Coalition for Homeless Veterans ${ }^{13}$ believes housing and employment opportunities are a top priority for homeless veterans.

\section{Complex deployment and reintegration needs}

Veteran issues related to separation from military service and other issues related to complex deployment needs are among specifically identified veteran issues. Veterans' successful reintegration into civilian life outcomes and interprofessional solutions stem from community involvement, access to resources, and support from peers. Reflection on best practices related particularly to employability 
and training builds on knowledge and skills gained in the military (ie, university accelerated programs for veterans where military medics and corpsmen transition through an accelerated program into nursing earning credit for military education and training [such as the Veterans Bachelor of Science in Nursing which is a Health Resources and Services Administration funded program]; Military Police to Criminal Justice, Navigational Experience and Knowledge to Geology and/or Geography, etc), employment post-military separation, reintegration into society, veteran demographics, homelessness prevention and other mental health and SUD programs that facilitate veterans' successful transition into urban civilian and family life. Successful reintegration after military separation is an essential focus for holistic and effective veteran care.

\section{Rationale for integrating veteran- centric content into curricula}

The veteran population is growing. In 2014, over 20 million veterans resided in the USA per the US Department of Veteran Affairs, 2015. ${ }^{14}$ Veterans are seeking health care services in the Veteran Health Administration as well as civilian treatment facilities. In order to understand and address health care needs of this vast and growing population, incorporating veteran-specific content into curricula is of primary importance. Veteran content specifically illuminates the unique yet complex health issues, mental and behavioral adjustment disorders, veteran wartime era, and civilian reintegration obstacles that, in combination, magnify their physical condition. Transparent presentation of the veteran circumstance can facilitate an interdisciplinary approach to care incorporating nursing, occupational therapy, physical therapy, mental health, pain management, nutrition, psychosocial, and social support services to ensure positive health care outcomes for this population. Several innovative strategies address these unique issues.

\section{Professional curricula}

Ideas for strategies to begin integration of veteran content into health care professional curricula include but are not limited to the following:

1. Provide presentations and seminars on veteran content delivered by well-known and distinguished speakers and/ or experts on particular veteran content.

2. Embed veteran content into courses (undergraduate and graduate) and identify specific courses to curriculum map where veteran content occurs.
3. Recruit and hire faculty that come from veteran and military backgrounds and experiences.

4. Provide faculty development opportunities to expand and improve their knowledge on veteran issues.

5. Identify veterans in clinical areas and provide clinical experiences for students with veteran patients of different war eras, branches of service, and military experience backgrounds.

6. Provide veteran simulations and case studies as part of undergraduate and graduate curriculum.

7. Provide students the opportunity to develop individualized patient plans and Subjective, Objective, Assessment, Plan (SOAP) notes that reflect needs of veteran patient.

The American Association of Colleges of Nursing ${ }^{15}$ offers a “Joining Forces: Enhancing Veterans' Care Tool Kit" (http://www.aacn.nche.edu/downloads/joining-forcestool-kit/educational-resources) with a variety of educational resources on many veteran issues, references to articles on particular veteran issues, case studies, a veteran assessment tool, curricular examples including slides and syllabi for veteran-specific courses. This site is an excellent resource.

\section{Discussion}

Currently, there are approximately 22 million US veterans. ${ }^{14}$ Preparing future health care providers to meet the needs of this extraordinary number of veterans is essential. Providing faculty development in the area of veteran-specific health issues and how to integrate veteran content into curricula will contribute to improving veteran outcomes and providing excellent care to those who served this country.

Total enrollees of veterans who utilize the VA health care system ( 8.9 million in 2013) ${ }^{16}$ is less than half the current total veteran population. Furthermore, approximately $61 \%$ of all separated $\mathrm{OEF} / \mathrm{OIF}$ veterans have used VA health care since October, 2001. ${ }^{17}$ This means that veterans are largely using civilian medical care facilities further stressing the need for health care providers to be well versed in veteran-specific health issues, war eras, and reintegration issues veterans face; in order to provide excellent veteran care and outcomes.

\section{Conclusion}

Promotion and implementation of veteran health issues into curricula, and other veteran content relevant to enhancing veteran care and outcomes, is essential in health care provider education and vital to the holistic care of veterans across the lifespan and across the country. Programs targeted at enhancing veteran-specific knowledge for faculty and 
students will serve to improve care for diverse veteran populations.

\section{Acknowledgments}

This project was supported by funds from the Division of Nursing and Public Health, Bureau of Health Workforce, Health Resources and Services Administration, Department of Health and Human Services Grant UF1HP26980, Veterans' Bachelor of Science Degree in Nursing Program (VBSN).

\section{Disclosure}

The authors have disclosed no conflicts of interest, financial or otherwise in this work.

\section{References}

1. Lattanzi JB, Purnell LD. Developing Cultural Competence in Physical Therapy Practice: 1st ed. Philadelphia, PA: FA Davis; 2005.

2. Fontana A, Rosenheck R. Treatment-seeking Veterans of Iraq and Afghanistan: Comparison with veterans of previous wars. J Nerv Ment Dis. 2008;196(7):513-521.

3. National Alliance on Mental Illness (NAMI). Depression and Veterans Fact Sheet. Arlington, VA: National Alliance on Mental Illness; 2009. Available from: http://www2.nami.org/Content/navigationMenu/ Mental_Illnesses/Depression/Depression_Veterans_Factsheet_2009. pdf. Accessed August 20, 2015.

4. LeardMann CA, Powell TM, Smith TC, et al. Risk factors associated with suicide in current and former US military personnel. JAMA. 2013; 310(5):496-506.

5. Johnson BS, Boudiab LD, Freundl M, Anthony M, Gmerek GB, Carter J. Enhancing veteran-centered care: a guide for nurses in non-VA setting. Am J Nurs. 2013;113(7):24-39.

6. American Psychiatric Association (APA) [homepage on the Internet]. Posttraumatic stress disorder. American Psychiatric Association; 2013. Available from: http://www.psychiatry.org/patients-families/ptsd/what-isptsd. Accessed May 28, 2015.

7. US Department of Veteran Affairs [homepage on the Internet]. Understanding traumatic brain injury. US Department of Veteran Affairs. Available from http://www.polytrauma.va.gov/understanding-tbi/. Accessed August 20, 2015.
8. Veterans Health Administration. Collaborative Care for Depression in the Primary Care Setting: A Primer on VA's Translating Initiatives for Depression into Effective Solutions (TIDES) Project. Veterans Health Administration; 2008. Available from http://www.hsrd.research.va.gov/ publications/internal/depression_primer.pdf. Accessed August 20, 2015.

9. Department of Veterans Affairs Mental Health Services Suicide Prevention Program. Kemp J, Bossarte R. Suicide Data Report, 2012. Department of Veterans Affairs Mental Health Services Suicide Prevention Program; 2012. Available from http://www.va.gov/opa/docs/ suicide-data-report-2012-final.pdf. Accessed August 20, 2015.

10. Federation of American Scientists. A Guide to Military Casualty Statistics: Operation Inherent Resolve, Operation New Dawn, Operation Iragi Freedom, and Operation Enduring Freedom. Congressional Research Service; 2015. Available from https://www.fas.org/sgp/crs/ natsec/RS22452.pdf. Accessed August 20, 2015.

11. US Department of Veteran Affairs [homepage on the Internet]. Military exposures. US Department of Veteran Affairs. Available from http:// www.publichealth.va.gov/exposures/. Accessed August 20, 2015.

12. Institute of Medicine. Veterans and Agent Orange: Update 2012. Washington, DC: National Academies Press; 2012.

13. National Coalition for Homeless Veterans (NCHV) [homepage on the Internet]. FAQ about homeless veterans. National Coalition for Homeless Veterans. Available from http://nchv.org/index.php/news/media/ background_and_statistics/.Accessed August 20, 2015.

14. US Department of Veteran Affairs. National Center for Veterans Analysis and Statistics. US Department of Veteran Affairs. Available from: http://www.va.gov/vetdata/docs/QuickFacts/Population_quickfacts. pdf. Accessed August 20, 2015.

15. American Academy of Colleges of Nursing (AACN) [homepage on the Internet]. Joining Forces: Enhancing Veterans' Care Tool Kit - Educational Resources. American Academy of Colleges of Nursing. Available from http://www.aacn.nche.edu/downloads/joining-forces-tool-kit/ educational-resources. Accessed August 20, 2015.

16. US Department of Veteran Affairs [homepage on the Internet]. Selected Veterans Health Administration Characteristics: FY2002 to FY2013. US Department of Veteran Affairs. Available from: http://www.va.gov/ vetdata/Utilization.asp. Accessed August 20, 2015.

17. US Department of Veteran Affairs [homepage on the Internet]. VA Health Care Utilization by recent veterans. US Department of Veteran Affairs. Available from: http://www.publichealth.va.gov/epidemiology/ reports/oefoifond/health-care-utilization/. Accessed August 20, 2015.
Advances in Medical Education and Practice

\section{Publish your work in this journal}

Advances in Medical Education and Practice is an international, peerreviewed, open access journal that aims to present and publish research on Medical Education covering medical, dental, nursing and allied health care professional education. The journal covers undergraduate education, postgraduate training and continuing medical education

\section{Dovepress}

including emerging trends and innovative models linking education, research, and health care services. The manuscript management system is completely online and includes a very quick and fair peer-review system. Visit http://www.dovepress.com/testimonials.php to read real quotes from published authors. 